\title{
A influência da hereditariedade na ocorrência de variáveis preditoras na gagueira crônica do desenvolvimento
}

\section{The influence of heredity on the predictor variables of chronic developmental stuttering}

\author{
Giovanna Cardoso Pinto ${ }^{\text {(D), Fabiola Juste }}{ }^{2}$ (1), Julia Biancalana Costa ${ }^{2}$ (1), Ana Paula Ritto ${ }^{1}$ (D), \\ Claudia Regina Furquim de Andrade ${ }^{2}$ (1)
}

\section{RESUMO}

Objetivo: Testar a variável da hereditariedade familiar para a gagueira crônica do desenvolvimento (GCD) como preditora de efeito direto no desfecho da fluência da fala em crianças. Métodos: Participaram do estudo 200 crianças, de 2 a 12 anos, de ambos os gêneros, sem distinção de raça e nível sócio-econômico-cultural, que apresentaram queixa de GCD, sem outras intercorrências de linguagem e/ou audição, no período de cinco anos. Os 200 participantes deste estudo foram divididos em três grupos (baixo risco para GCD, médio risco para GCD e alto risco para GCD) conforme os indicadores de risco aferidos pelo Protocolo de risco para a gagueira do desenvolvimento. Para determinação da variável de controle (hereditariedade positiva para a gagueira) foi considerado afetado o participante que apresentava familiar de primeiro grau (pai, mãe, irmãos) que se auto identificava como pessoa com gagueira. Todos os participantes foram avaliados segundo o Protocolo de risco para a gagueira do desenvolvimento e pela Avaliação do Perfil da Fluência de Fala. Resultados: Os grupos de baixo, médio e alto risco para GCD com hereditariedade positiva não se diferenciaram estatisticamente dos grupos de baixo, médio e alto risco para GCD com hereditariedade negativa para nenhuma das variáveis demográficas e resultado da análise do Perfil de Fluência da Fala. Conclusão: A variável hereditariedade não indicou o grau de risco na manifestação da fala nem identificou, decisivamente, as crianças em risco de persistência para a GCD.

Palavras-chave: Fonoaudiologia; Gagueira; Genética; Hereditariedade; Crianças

\begin{abstract}
Purpose: To test if the variable family heredity for chronic developmental stuttering (CDS) is a direct predictor of the speech fluency outcome in children. Methods: Participants of the study were 200 children, between 2 and 12 years of age, of both genders, with no racial and socioeconomic distinction, diagnosed with a complaint of CDS, and with no language and/ or hearing comorbidity, over a period of 5 years. Participants were divided in three study groups (low risk for CDS, moderate risk for CDS, and high risk for CDS) according to the risk indicators determined by the Risk Protocol for Developmental Stuttering. In order to determine the control variable (positive heredity for stuttering), we considered the participant as being affected if he/she presented a first-degree family member (father, mother, siblings) who self-declared themselves as a person who stuttered. All of the participants were assessed according to Risk Protocol for Developmental Stuttering and to The Speech Fluency Profile Assessment. Results: No significant difference was observed for the demographic variables and for the results on The Fluency Profile Assessment among the groups with mild, moderate and high risk of stuttering when comparing the groups with positive and negative family heredity. Conclusion: The variable family heredity did not indicate the risk level for the manifestation of stuttering and also did not identify those at risk of presenting CDS.
\end{abstract}

Keywords: Speech, Language and Hearing Sciences; Stuttering; Genetics; Heredity; Children

\footnotetext{
Trabalho realizado no Laboratório de Investigação Fonoaudiológica em Fluência, Funções da Face e Disfagia, Departamento de Fisioterapia, Fonoaudiologia e Terapia Ocupacional, Faculdade de Medicina, Universidade de São Paulo - USP - São Paulo (SP), Brasil.

${ }^{1}$ Hospital das Clínicas, Faculdade de Medicina, Universidade de São Paulo - USP - São Paulo (SP), Brasil.

${ }^{2}$ Curso de Fonoaudiologia, Departamento de Fisioterapia, Fonoaudiologia e Terapia Ocupacional, Faculdade de Medicina, Universidade de São Paulo - USP São Paulo (SP), Brasil.

Conflito de interesses: Não.

Contribuição dos autores: GCP responsável coleta, tabulação, análise dos dados da pesquisa e elaboração do artigo; FJ responsável pela análise dos dados; colaborou com a revisão final do artigo; JBC colaborou tabulação e análise dos dados da pesquisa; APR realizou o tratamento estatístico dos dados e a interpretação dos resultados; CRFA responsável pela concepção e delineamento do estudo; pela interpretação dos dados; pela orientação das etapas de execução da pesquisa e elaboração e revisão final do artigo.

Financiamento: CNPq na forma de bolsa de Produtividade em Pesquisa (processo no 305860/2018-6) e Iniciação Científica (processo nº 157266/2017-6).

Autor correspondente: Claudia Regina Furquim de Andrade. E-mail: clauan@usp.br
}

Recebido: Fevereiro 11, 2021; Aceito: Julho 11, 2021 


\section{INTRODUÇÃO}

A gagueira crônica do desenvolvimento (GCD) é um distúrbio caracterizado por rupturas involuntárias no fluxo suave e contínuo da fala. Seu surgimento ocorre, frequentemente, durante a primeira infância (dos 2 aos 4 anos de idade), podendo, entretanto, manifestar-se mais tardiamente, até os 12 anos de idade $^{(1-6)}$. Mundialmente, as taxas de incidência variam de 5\% a $18 \%{ }^{(4)}$ e a prevalência, aproximadamente $1 \%{ }^{(5)}$. Os principais sintomas de fala da GCD são os bloqueios, as repetições de sons e sílabas e os prolongamentos inadequados dos sons. Estas tipologias de rupturas podem ser, ou não, acompanhadas de tensão física ${ }^{(1-6)}$.

Há várias décadas, diversas hipóteses foram levantadas quanto à etiologia da GCD. Com o advento das técnicas de neuroimagem, surgiu uma mudança de paradigma, implicando fatores neurológicos anatômicos e funcionais para explicar não somente a origem, mas a persistência da $\mathrm{GCD}^{(7-12)}$.

Em 2015, Chang et al. ${ }^{(10)}$ realizaram um estudo de neuroimagem que analisou as atividades e conectividades estruturais em áreas específicas do cérebro, indicando que, em crianças com GCD, foi observada redução do desenvolvimento da substância branca na região motora oral esquerda e redução do desenvolvimento da substância cinzenta na região frontal inferior esquerda (área de Broca). Os autores concluíram que estas modificações cerebrais estruturais acarretam uma redução da funcionalidade e da conectividade no circuito núcleos da base, tálamo e córtex. Essa rede responde pelo controle motor dos movimentos independentes, como os da fala. Os resultados também indicaram redução da conectividade entre as redes, envolvendo as interações auditiva e motoras (giro temporal, superior, posterior esquerdo, ínsula, área motora suplementar e giro frontal superior).

Posteriormente, em 2018, Chang et al. ${ }^{(11)}$ analisaram a arquitetura das redes neurais do cérebro em repouso de crianças com GCD. Para isso, os autores reuniram um grande conjunto longitudinal de dados de neuroimagem que incluiram crianças com GCD e crianças fluentes, realizando uma análise de rede de todo o cérebro, para investigar as mudanças de conectividade intra e inter-rede associadas à gagueira. Os resultados indicaram que as diferenças da arquitetura neural das crianças com GCD apresentadas no estudo de $2015^{(10)}$ têm impacto na conectividade com as redes que dão suporte às habilidades de atenção, motricidade e propiocepção, o que não apenas coloca as crianças em risco de desenvolver gagueira, como pode influenciar sua persistência ou recuperação.

Estudo realizado em $2018^{(12)}$ teve como objetivo analisar as medidas morfométricas cerebrais em crianças com gagueira, divididas em dois grupos: crianças que apresentavam gagueira persistente e crianças que se recuperaram da gagueira sem qualquer tipo de intervenção terapêutica. Os resultados indicaram que o grupo de crianças com gagueira persistente se diferenciou dos grupos de crianças com gagueira recuperada e do grupo controle de crianças fluentes, apresentando redução da espessura nas regiões corticais motora esquerda e prémotora lateral. Estes resultados forneceram fortes evidências de um deficit primário na rede de fala do hemisfério esquerdo, especificamente envolvendo córtex pré-motor lateral e córtex motor primário nos casos de GCD. Os autores ainda apontaram para um possível mecanismo compensatório envolvendo o córtex pré-motor medial esquerdo em indivíduos que se recuperam da gagueira na infância.

Outra grande corrente de estudos apoia a existência de uma base genética na etiologia da $\mathrm{GCD}^{(13-23)}$. Estudos realizados com famílias de indivíduos com GCD têm demonstrado que esses indivíduos, quando comparados à um grupo controle de indivíduos fluentes, são mais propensos a ter membros da família que também relatam um histórico de gagueira( ${ }^{(13,17-19)}$. Os estudos estimaram que, entre $30 \%$ e $60 \%$ dos indivíduos com GCD, tinham um histórico familiar positivo, enquanto no grupo controle, essa ocorrência foi menor que $10 \%$.

A GCD tem sido associada a alterações nos cromossomos $9,10,12,13$ e $18^{(18,19)}$. A análise genética do gene DRD2, um receptor de dopamina presente no cérebro, mostrou maior frequência deste alelo em indivíduos com $\mathrm{GCD}^{(20)}$, porém, este achado não foi replicado em outro estudo compatível subsequente $^{(21)}$. As funções relacionadas aos genes identificados incluem neurometabolismo, interação intercelular, regulação da transcrição embrionária e modificação de comportamento ${ }^{(19-21)}$.

Frigerio-Domingues et al ${ }^{(13)}$ relataram que estudos realizados com indivíduos com GCD e seus familiares identificaram uma série de loci de ligação genética altamente significativa, sugerindo a existência de genes causadores em locais cromossômicos específicos. Além disso, estes estudos identificaram mutações nos genes GNPTAB, GNPTG, NAGPA e AP4E1 associados à $\mathrm{GCD}$. Segundo os autores ${ }^{(13)}$, estes quatro genes estão intimamente relacionados funcionalmente e envolvidos no processo de tráfico intracelular. De acordo com os autores ${ }^{(13)}$, deficits nesta função celular são agora reconhecidos como causadores de uma ampla gama de distúrbios neurológicos. Estudos $^{(24,25)}$ descreveram que variantes genéticas específicas no GNPTAB, GNPTG e NAGPA (todas relacionadas a processos lisosômicos e conhecidas por causar mucolipidose tipo II e III mutações homozigosas autossômicas) estão especificamente ligadas aos casos de GCD.

Os fundamentos neurobiológicos da GCD permanecem incertos. Embora as pesquisas envolvendo esta temática tenham proporcionado aos pesquisadores a capacidade de identificar vários perfis genéticos associados ao distúrbio ${ }^{(13-23)}$, o modo como essas origens genéticas específicas impactam circuitos neuronais e como eles geram ou facilitam o surgimento da GCD ainda é desconhecido.

Um objetivo comum entre os pesquisadores da GCD tem sido identificar preditores que possam indicar um prognóstico mais assertivo quanto a eficácia do processo terapêutico ${ }^{(13)}$. Dois estudos $^{(24,25)}$ procuraram identificar preditores de recuperação de crianças pequenas que gaguejam. Entre as múltiplas variáveis investigadas, idade, gênero e hereditariedade familiar para o distúrbio têm sido identificadas de forma relativamente consistente, isoladas ou em combinação, como preditoras da GCD. Os resultados indicaram que existe maior recuperação entre meninas e, principalmente, em meninas mais novas (antes dos 4 anos de idade) e maior recuperação na ausência de histórico de hereditariedade familiar de GCD, independente do gênero e da idade. Conforme ampla literatura ${ }^{(18-29)}$, o histórico familiar positivo para a gagueira é uma variável de associação independente, ou seja, transmite a susceptibilidade para a gagueira. Dentre as variáveis de risco (características e/ou circunstâncias que estão relacionadas com o aumento da probabilidade de ocorrência da gagueira), há variáveis familiares, comportamentais e sociais ${ }^{(1,3,4,6,24,25)}$. 
Diante do exposto, o objetivo desta pesquisa foi testar a variável da hereditariedade familiar para a GCD como preditora de efeito direto no desfecho da fluência da fala em crianças. A variável de hereditariedade foi testada nos diferentes graus de risco para a GCD.

\section{MÉTODOS}

A pesquisa realizada se configura como um estudo de caso controle, cuja variável controle é a hereditariedade para a gagueira crônica do desenvolvimento (GCD). As variáveis analisadas foram: disfluências comuns, disfluências gagas e velocidade de fala. O estudo foi aprovado pelo Comitê de Ética em Pesquisa do Hospital das Clínicas da Faculdade de Medicina da Universidade de São Paulo - CAPPesq, no 2.235.170). Os dados foram coletados a partir do banco de dados da Divisão de Fonoaudiologia do Hospital das Clínicas da Faculdade de Medicina da Universidade de São Paulo, dispensando, assim, a obrigatoriedade de assinatura do Termo de Consentimento Livre e Esclarecido. A pesquisa foi considerada de baixo risco, sendo assegurada a continuidade da assistência fonoaudiológica para os participantes. A pesquisa recebeu fomento do Conselho Nacional de Desenvolvimento Científico e Tecnológico CNPq, na forma de bolsa de Produtividade em Pesquisa (processo no 305860/2018-6) e Iniciação Científica (processo no $157266 / 2017-6)$.

\section{Participantes}

Participaram do estudo 200 crianças, de 2 a 12 anos, de ambos os gêneros, sem distinção de raça e sem restrição de nível socioeconômico-cultural, que apresentaram queixa de GCD, sem outras intercorrências de linguagem e/ou audição, no período de cinco anos. Os 200 participantes foram divididos em três grupos (baixo risco, médio e alto), conforme os indicadores de risco para a GCD.

O desenho da elegibilidade dos participantes é apresentado na Figura 1.

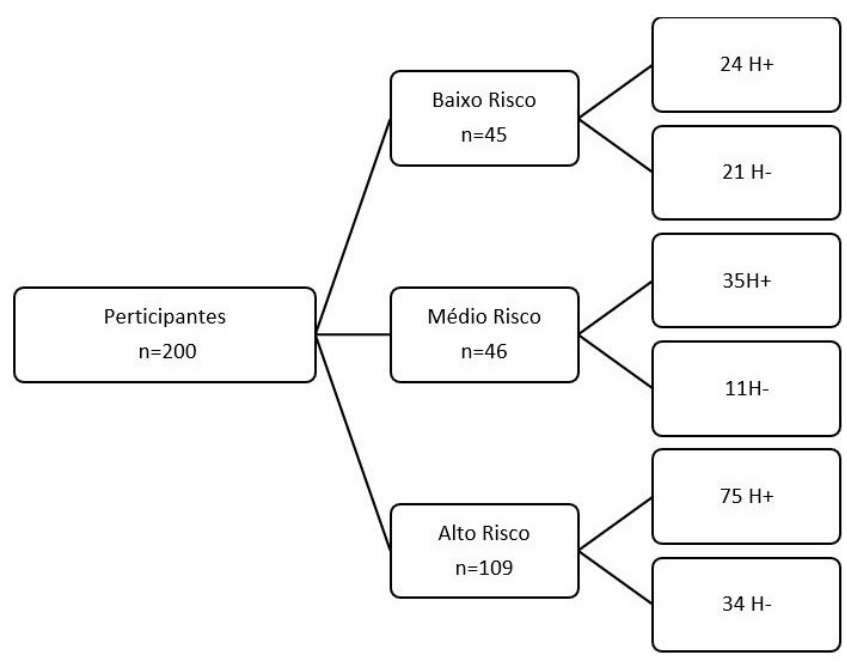

Figura 1. Fluxograma de elegibilidade

Legenda: $\mathrm{n}=$ número de sujeitos; $\mathrm{H}=$ hereditariedade

\section{Material e procedimento}

Todos os participantes foram avaliados pelos escores de risco para GCD segundo o Protocolo de Risco para a Gagueira do Desenvolvimento (PRGD ${ }^{(26)}$ ) e pelo Perfil da Fluência de $\mathrm{Fala}^{(27)}$. Para a composição dos grupos de risco para gagueira, foram consideradas variáveis de risco: tempo de surgimento da gagueira; tipo de surgimento; fatores linguísticos associados; fatores qualitativos associados; componentes estressantes da qualidade de vida; histórico mórbido pré-natal; reação familiar para o distúrbio; reação da própria criança para o distúrbio e reação social (família estendida e escola) ao distúrbio. Foram consideradas participantes de baixo risco as crianças que apresentaram até $33 \%$ de indicadores de risco. Foram consideradas de risco médio as crianças que apresentaram entre 34 e $66 \%$ de indicadores de risco. Foram consideradas de alto risco as crianças que apresentaram acima de $67 \%$ de indicadores de risco.

Para determinação da variável de controle - hereditariedade positiva para a gagueira - foi considerado afetado o participante que apresentava familiar de primeiro grau (pai, mãe, irmãos) que se autoidentificava como pessoa com gagueira, com confirmação pela percepção familiar.

Quanto à Avaliação do Perfil da Fluência da Fala ${ }^{(27)}$, foram analisadas amostras de fala espontânea, obtidas a partir de figura estímulo, contabilizando 200 sílabas fluentes para cada um dos participantes. As amostras foram analisadas de acordo com as variáveis do Perfil da Fluência da Fala, sendo: disfluências comuns (hesitações, interjeições, repetição de palavras, segmentos e frases, revisões e palavras não terminadas); disfluências gagas (repetição de sons e sílabas, bloqueios, prolongamentos, pausas e intrusões de sons ou segmentos); velocidade de fala (em palavras e sílabas por minuto).

\section{Análise dos dados}

O estudo foi realizado com cegamento das transcrições das amostras de fala, para evitar a má interpretação dos resultados do teste, evitando vieses, preconceitos e demais informações que pudessem afetar o julgamento no momento de transcrição das amostras. Para isso, as transcrições e análises foram realizadas por fonoaudiólogos que não participaram do processo de avaliação dos participantes da pesquisa.

Para garantia do índice de confiabilidade das transcrições das amostras de fala, foram convocados três juízes fonoaudiólogos, com experiência na área, para avaliação da acurácia das transcrições. Para tanto, foram sorteadas as transcrições de $30 \%$ das amostras e o índice de concordância entre os juízes obteve, no mínimo, $85 \%$ de confiabiliade;

Os dados coletados foram submetidos à análise estatística no software IBM-SPSS, versão 26. Os dados quantitativos receberam análise descritiva (média e desvio padrão) e inferencial, comparando os grupos (teste t de Student para dados com distribuição paramétrica e teste de Mann-Whitney para dados com distribuição não paramétrica). Os dados qualitativos receberam análise descritiva (contagem total e porcentagem) e inferencial, comparando os grupos (teste Qui-Quadrado de Pearson). O nível de significância adotado em todas as análises foi de $5 \%$. 


\section{RESULTADOS}

Os participantes do grupo de baixo risco foram comparados de acordo com suas variáveis demográficas e resultado da análise do Perfil de Fluência da Fala. Não foram encontradas diferenças significativas entre os grupos para nenhuma das variáveis (Tabela 1 ).

Os participantes do grupo de risco médio foram comparados de acordo com suas variáveis demográficas e resultado da análise do Perfil de Fluência da Fala. Foram encontradas diferenças significativas entre os grupos apenas para as variáveis idade e repetição de segmentos (Tabela 2).

Os participantes do grupo de alto risco foram comparados de acordo com suas variáveis demográficas e resultado da análise do Perfil de Fluência da Fala. Não foram encontradas diferenças significativas entre os grupos para nenhuma das variáveis (Tabela 3 ).

Os 200 participantes deste estudo, divididos em dois grupos, com hereditariedade positiva e sem hereditariedade,

Tabela 1. Comparação entre os participantes do grupo de baixo risco

\begin{tabular}{|c|c|c|c|c|}
\hline & & $\begin{array}{l}\text { Hereditariedade } \\
\text { Positiva }(n=24) \\
\end{array}$ & $\begin{array}{c}\text { Sem Hereditariedade } \\
(\mathrm{n}=21)\end{array}$ & Valor de $p$ \\
\hline \multirow{2}{*}{$\begin{array}{l}\text { Gênero } \\
\text { Número total (porcentagem) }\end{array}$} & Masculino & $12(50,0 \%)$ & $14(66,7 \%)$ & 0,259 \\
\hline & Feminino & $12(50,0 \%)$ & $7(33,3 \%)$ & \\
\hline $\begin{array}{l}\text { Idade, em anos } \\
\text { Média ( } \pm \text { desvio padrão) }\end{array}$ & & $3,8( \pm 1,8)$ & $4,7( \pm 2,8)$ & 0,394 \\
\hline \multirow{7}{*}{$\begin{array}{l}\text { Disfluências Comuns } \\
\text { Média ( } \pm \text { desvio padrão) }\end{array}$} & Hesitações & $4,8( \pm 11,1)$ & $2,1( \pm 2,6)$ & 0,887 \\
\hline & Interjeições & $0,7( \pm 1,1)$ & $0,9( \pm 1,3)$ & 0,670 \\
\hline & Revisões & $1,4( \pm 1,6)$ & $0,9( \pm 1,2)$ & 0,323 \\
\hline & Pal. não terminadas & $0,6( \pm 0,7)$ & $0,4( \pm 1,0)$ & 0,175 \\
\hline & Repetição de palavras & $5,3( \pm 5,6)$ & $4,6( \pm 3,8)$ & 0,855 \\
\hline & Repetição de segmentos & $1,4( \pm 1,7)$ & $1,1( \pm 1,4)$ & 0,538 \\
\hline & Repetição de frases & $0,1( \pm 0,3)$ & $0,0( \pm 0,0)$ & 0,181 \\
\hline \multirow{6}{*}{$\begin{array}{l}\text { Disfluências Gagas } \\
\text { Média ( } \pm \text { desvio padrão) }\end{array}$} & Repetição de sílabas & $2,0( \pm 2,3)$ & $2,4( \pm 2,8)$ & 0,779 \\
\hline & Repetição de sons & $1,9( \pm 3,3)$ & $0,9( \pm 1,7)$ & 0,228 \\
\hline & Prolongamentos & $1,9( \pm 3,8)$ & $2,2( \pm 4,1)$ & 0,932 \\
\hline & Bloqueios & $1,7( \pm 3,5)$ & $0,3( \pm 0,6)$ & 0,142 \\
\hline & Pausas & $0,2( \pm 0,5)$ & $0,1( \pm 0,4)$ & 0,908 \\
\hline & Intrusão & $0,1( \pm 0,3)$ & $0,5( \pm 1,8)$ & 0,504 \\
\hline \multirow{2}{*}{$\begin{array}{l}\text { Velocidade de fala } \\
\text { Média ( } \pm \text { desvio padrão) }\end{array}$} & Palavras por minuto & $75,1( \pm 27,5)$ & $82,3( \pm 25,3)$ & 0,413 \\
\hline & Sílabas por minuto & $123,9( \pm 47,5)$ & $137,0( \pm 50,6)$ & 0,474 \\
\hline
\end{tabular}

Legenda: $\mathrm{n}=$ número de sujeitos; Pal. = palavras

Tabela 2. Comparação entre os participantes do grupo de risco médio

\begin{tabular}{|c|c|c|c|c|}
\hline & & $\begin{array}{l}\text { Hereditariedade } \\
\text { Positiva }(n=35)\end{array}$ & $\begin{array}{l}\text { Sem Hereditariedade } \\
(\mathbf{n}=11)\end{array}$ & Valor de $p$ \\
\hline \multirow{2}{*}{$\begin{array}{l}\text { Gênero } \\
\text { Número total (porcentagen }\end{array}$} & Masculino & $28(80,0 \%)$ & $7(63,6 \%)$ & 0,267 \\
\hline & Feminino & $7(20,0 \%)$ & $4(36,4 \%)$ & \\
\hline \multicolumn{2}{|l|}{$\begin{array}{l}\text { Idade, em anos } \\
\text { Média ( } \pm \text { desvio padrão) }\end{array}$} & $5,9( \pm 1,6)$ & $4,7( \pm 1,5)$ & $0,027^{*}$ \\
\hline \multirow{7}{*}{$\begin{array}{l}\text { Disfluências Comuns } \\
\text { Média ( } \pm \text { desvio padrão) }\end{array}$} & Hesitações & $3,7( \pm 4,4)$ & $2,9( \pm 3,3)$ & 0,780 \\
\hline & Interjeições & $1,3( \pm 2,2)$ & $0,6( \pm 1,2)$ & 0,509 \\
\hline & Revisões & $1,2( \pm 1,8)$ & $0,4( \pm 0,5)$ & 0,075 \\
\hline & Pal. não terminadas & $0,5( \pm 1,1)$ & $0,6( \pm 1,5)$ & 0,939 \\
\hline & Repetição de palavras & $3,9( \pm 3,8)$ & $6,6( \pm 6,7)$ & 0,117 \\
\hline & Repetição de segmentos & $0,9( \pm 1,4)$ & $2,1( \pm 1,6)$ & $0,014^{*}$ \\
\hline & Repetição de frases & $0,1( \pm 0,5)$ & $0,0( \pm 0,0)$ & 0,780 \\
\hline \multirow{6}{*}{$\begin{array}{l}\text { Disfluências Gagas } \\
\text { Média ( } \pm \text { desvio padrão) }\end{array}$} & Repetição de sílabas & $1,6( \pm 3,1)$ & $2,0( \pm 2,9)$ & 0,284 \\
\hline & Repetição de sons & $1,3( \pm 1,8)$ & $0,6( \pm 0,9)$ & 0,251 \\
\hline & Prolongamentos & $2,3( \pm 2,1)$ & $2,8( \pm 3,8)$ & 0,780 \\
\hline & Bloqueios & $2,2( \pm 3,9)$ & $4,2( \pm 6,5)$ & 0,999 \\
\hline & Pausas & $1,0( \pm 2,6)$ & $1,3( \pm 1,8)$ & 0,296 \\
\hline & Intrusão & $0,0( \pm 0,0)$ & $0,2( \pm 0,6)$ & 0,666 \\
\hline \multirow{2}{*}{$\begin{array}{l}\text { Velocidade de fala } \\
\text { Média ( } \pm \text { desvio padrão) }\end{array}$} & Palavras por minuto & $63,2( \pm 29,5)$ & $62,7( \pm 28,3)$ & 0,960 \\
\hline & Sílabas por minuto & $115,2( \pm 52,9)$ & $106,8( \pm 50,1)$ & 0,761 \\
\hline
\end{tabular}

*Diferença significativa de acordo com o teste de Mann-Whitney

Legenda: $\mathrm{n}=$ número de sujeitos; Pal. = palavras 
foram comparados de acordo com suas variáveis demográficas e resultado da análise do Perfil de Fluência da Fala. Não foram encontradas diferenças significativas entre os grupos (Tabela 4).

\section{DISCUSSÃO}

A GCD é um distúrbio complexo e heterogêneo. Há consenso na literatura que a inter-relação dos processos linguísticos,

Tabela 3. Comparação entre os participantes do grupo de alto risco

\begin{tabular}{|c|c|c|c|c|}
\hline & & $\begin{array}{l}\text { Hereditariedade } \\
\text { Positiva }(n=75)\end{array}$ & $\begin{array}{l}\text { Sem Hereditariedade } \\
(\mathrm{n}=39)\end{array}$ & Valor de p \\
\hline \multirow{2}{*}{$\begin{array}{l}\text { Gênero } \\
\text { Número total (porcentagem) }\end{array}$} & Masculino & $55(73,3 \%)$ & $21(61,8 \%)$ & 0,223 \\
\hline & Feminino & $20(26,7 \%)$ & $13(38,2 \%)$ & \\
\hline $\begin{array}{l}\text { Idade, em anos } \\
\text { Média ( } \pm \text { desvio padrão) }\end{array}$ & & $7,6( \pm 2,1)$ & $8,2( \pm 2,4)$ & 0,163 \\
\hline \multirow{7}{*}{$\begin{array}{l}\text { Disfluências Comuns } \\
\text { Média ( } \pm \text { desvio padrão) }\end{array}$} & Hesitações & $4,3( \pm 4,3)$ & $4,6( \pm 4,0)$ & 0,540 \\
\hline & Interjeições & $1,8( \pm 2,9)$ & $1,4( \pm 2,1)$ & 0,706 \\
\hline & Revisões & $1,4( \pm 1,6)$ & $1,7( \pm 2,0)$ & 0,674 \\
\hline & Pal. não terminadas & $0,6( \pm 1,1)$ & $0,4( \pm 0,7)$ & 0,553 \\
\hline & Repetição de palavras & $5,5( \pm 5,4)$ & $4,4( \pm 3,7)$ & 0,488 \\
\hline & Repetição de segmentos & $1,3( \pm 1,7)$ & $1,5( \pm 1,7)$ & 0,745 \\
\hline & Repetição de frases & $0,1( \pm 0,3)$ & $0,1( \pm 0,3)$ & 0,508 \\
\hline \multirow{6}{*}{$\begin{array}{l}\text { Disfluências Gagas } \\
\text { Média ( } \pm \text { desvio padrão) }\end{array}$} & Repetição de sílabas & $2,2( \pm 2,5)$ & $2,0( \pm 2,5)$ & 0,579 \\
\hline & Repetição de sons & $1,8( \pm 2,8)$ & $1,9( \pm 3,3)$ & 0,517 \\
\hline & Prolongamentos & $3,8( \pm 4,3)$ & $3,5( \pm 4,9)$ & 0,393 \\
\hline & Bloqueios & $4,3( \pm 5,8)$ & $4,4( \pm 7,5)$ & 0,104 \\
\hline & Pausas & $0,9( \pm 1,8)$ & $0,4( \pm 0,8)$ & 0,228 \\
\hline & Intrusão & $0,8( \pm 2,2)$ & $1,3( \pm 4,3)$ & 0,727 \\
\hline \multirow{2}{*}{$\begin{array}{l}\text { Velocidade de fala } \\
\text { Média ( } \pm \text { desvio padrão) }\end{array}$} & Palavras por minuto & $69,4( \pm 24,9)$ & $75,0( \pm 26,1)$ & 0,151 \\
\hline & Sílabas por minuto & $115,3( \pm 44,3)$ & $130,7( \pm 51,5)$ & 0,099 \\
\hline
\end{tabular}

Legenda: $\mathrm{n}=$ número de sujeitos; Pal. = palavras

Tabela 4. Comparação entre os grupos com hereditariedade positiva versus sem hereditariedade - para todos os participantes do estudo

\begin{tabular}{|c|c|c|c|c|}
\hline & & $\begin{array}{l}\text { Hereditariedade } \\
\text { Positiva }(n=134)\end{array}$ & $\begin{array}{l}\text { Sem Hereditariedade } \\
(n=66)\end{array}$ & Valor de $p$ \\
\hline \multirow{2}{*}{$\begin{array}{l}\text { Gênero } \\
\text { Número total (porcentagem) }\end{array}$} & Masculino & $95(70,9 \%)$ & $42(63,6 \%)$ & \multirow[t]{2}{*}{0,299} \\
\hline & Feminino & $39(29,1 \%)$ & $24(36,4 \%)$ & \\
\hline $\begin{array}{l}\text { Idade, em anos } \\
\text { Média ( } \pm \text { desvio padrão) }\end{array}$ & & $6,4( \pm 2,4)$ & $6,5( \pm 2,9)$ & 0,944 \\
\hline \multirow{7}{*}{$\begin{array}{l}\text { Disfluências Comuns } \\
\text { Média ( } \pm \text { desvio padrão) }\end{array}$} & Hesitações & $4,2( \pm 6,1)$ & $3,5( \pm 3,6)$ & 0,303 \\
\hline & Interjeições & $1,5( \pm 2,5)$ & $1,1( \pm 1,7)$ & 0,224 \\
\hline & Revisões & $1,3( \pm 1,6)$ & $1,2( \pm 1,7)$ & 0,579 \\
\hline & Pal. não terminadas & $0,6( \pm 1,1)$ & $0,5( \pm 0,9)$ & 0,360 \\
\hline & Repetição de palavras & $5,1( \pm 5,1)$ & $4,8( \pm 4,3)$ & 0,751 \\
\hline & Repetição de segmentos & $1,2( \pm 1,6)$ & $1,4( \pm 1,6)$ & 0,400 \\
\hline & Repetição de frases & $0,1( \pm 0,4)$ & $0,1( \pm 0,2)$ & 0,356 \\
\hline \multirow{6}{*}{$\begin{array}{l}\text { Disfluências Gagas } \\
\text { Média ( } \pm \text { desvio padrão) }\end{array}$} & Repetição de sílabas & $2,0( \pm 2,6)$ & $2,1( \pm 2,6)$ & 0,744 \\
\hline & Repetição de sons & $1,7( \pm 2,7)$ & $1,4( \pm 2,6)$ & 0,406 \\
\hline & Prolongamentos & $3,1( \pm 3,8)$ & $3,0( \pm 4,5)$ & 0,871 \\
\hline & Bloqueios & $3,3( \pm 5,1)$ & $3,0( \pm 6,2)$ & 0,800 \\
\hline & Pausas & $0,8( \pm 1,9)$ & $0,4( \pm 1,0)$ & 0,101 \\
\hline & Intrusão & $0,5( \pm 1,7)$ & $0,9( \pm 3,3)$ & 0,398 \\
\hline \multirow{2}{*}{$\begin{array}{l}\text { Velocidade de fala } \\
\text { Média ( } \pm \text { desvio padrão) }\end{array}$} & Palavras por minuto & $68,8( \pm 26,7)$ & $75,2( \pm 26,6)$ & 0,112 \\
\hline & Sílabas por minuto & $116,8( \pm 47,0)$ & $128,7( \pm 51,2)$ & 0,115 \\
\hline
\end{tabular}

Legenda: $\mathrm{n}=$ número de sujeitos; Pal. = palavras 
motores, sensoriais e emocionais, estão envolvidos em seu desenvolvimento e/ou manutenção ${ }^{(1-3,5,6,10-12,24,25)}$.

É de interesse prático e teórico identificar os fatores que possam estar associados ao aumento das chances de recuperação espontânea da gagueira ou da sua cronicidade. Até o momento, o risco de persistência do distúrbio é atribuído ao histórico familiar positivo para a gagueira persistente ${ }^{(12,13,15,19,22)}$. O marcador de gênero - foco no genêro masculino - também é considerado fator positivo para a gagueira persistente, assim como a idade da criança e o tempo de surgimento do distúrbio ${ }^{(3,5,13,15-19)}$.

Os resultados obtidos na pesquisa, na forma como foi realizada, isolamento da variável hereditariedade, não indicaram a ocorrência de variáveis de fala que pudessem ser preditoras da gagueira. A hipótese deste estudo seria que a hereditariedade positiva poderia supor, de algum modo, que haveria uma sintomatologia de rupturas indicativa de alto risco para a cronicidade. Não foi o encontrado, a hereditariedade não indica o grau de risco na manifestação da fala nem identifica, decisivamente, as crianças em risco de persistência.

Embora a literatura evidencie que há um componente genético para a transmissão da suscetibilidade à gagueira ${ }^{(13-23)}$, a relação entre o componente genético com a gagueira - a persistência, a recuperação e a sintomatologia de fala - permanece incerta.

Como o fator genético ainda não foi isolado em relação ao risco do distúrbio (baixo, médio ou alto) há uma significativa dificuldade em priorizar os procedimentos terapêuticos mais indicados em cada caso. Atrasar a entrada em terapia fonoaudiológica em pré-escolares de risco pode favorecer a instalação dos traços secundários da gagueira - desenvolver sentimentos e atitudes negativas em relação à comunicação(16,28-30). Variáveis clinicamente relevantes - tipologia de rupturas, tempo de surgimento dos sintomas, tempo de permanência dos sintomas e reações pessoais e familiares devem ser consideradas. Estabelecer práticas terapêuticas competentes para cada grupo de risco ainda é a medida mais segura para o atendimento às crianças. A intervenção precoce - indireta e/ou direta - adequada, que permita à criança manter níveis reduzidos de rupturas de fala associadas à tensão articulatória, bem como apoio emocional e educacional à ela e à família são recursos desejáveis ${ }^{(16)}$.

Quanto à variável genética, até o momento não foi isolada a forma de transmissão ${ }^{(13-23)}$. A recuperação da GCD não parece ser uma forma geneticamente mais branda de gagueira, nem os dois tipos de gagueira parecem ser distúrbios geneticamente independentes $^{(15-19)}$. Os dados são mais consistentes com a hipótese de que a gagueira persistente e a recuperada possuem etiologia genética comum e que a persistência é, em parte, devido a fatores genéticos adicionais ${ }^{(15-19)}$.

Embora haja consenso na literatura ${ }^{(1-6)}$ que a ruptura de fala do tipo bloqueio (reação motora interna de quebra temporal do som e/ou sílaba) como característica saliente da gagueira, o resultado da pesquisa aqui apresentada não encontrou diferença significativa, quer na forma, quer na quantidade de rupturas desse tipo em crianças com ou sem hereditariedade positiva para a GCD.

\section{CONCLUSÃO}

O estudo não identificou sintomas de fala que pudessem ser considerados como parte da atribuição do fenótipo de gagueira crônica do desenvolvimento, ou seja, crianças com e sem antecedentes genéticos para a gagueira não se diferenciaram quanto às variáveis testadas.

\section{AGRADECIMENTOS}

Ao CNPq pelo financiamento desta pesquisa na forma de duas bolsas: Produtividade em pesquisa nível 1A (processo no 305860/2018-6) e Iniciação Científica (processo no 157266/2017-6).

\section{REFERÊNCIAS}

1. Andrade, CRF. Abordagem neurolinguística e motora da gagueira. In: Fernandes FDM, Mendes BCA, Navas ALPG, editores. Tratado de Fonoaudiologia. 2. ed. São Paulo: Roca; 2010. p. 423-33.

2. Craig-McQuaide A, Akram H, Zrinzo L, Tripoliti E. A review of brain circuitries involved in stuttering. Front Hum Neurosci. 2014;8:884. http://dx.doi.org/10.3389/fnhum.2014.00884. PMid:25452719.

3. Yairi E, Ambrose N. Epidemiology of stuttering: 21 st century advances. J Fluency Disord. 2013;38(2):66-87. http://dx.doi.org/10.1016/j. jfludis.2012.11.002. PMid:23773662.

4. Reilly S, Onslow M, Packman A, Wake M, Bavin EL, Prior M, et al. Predicting stuttering onset by the age of 3 years: a prospective, community cohort study. Pediatrics. 2009;123(1):270-7. http://dx.doi. org/10.1542/peds.2007-3219. PMid:19117892.

5. Bloodstein O, Bernstein-Ratner N. A handbook of stuttering. Clifton Park: Thomson Delmar Learning; 2008.

6. Benito-Aragón C, Gonzalez-Sarmiento R, Liddell T, Diez I, d'Oleire Uquillas F, Ortiz-Terán L, et al. Neurofilament-lisosomal genetic intersections in the cortical network of stuttering. Prog Neurobiol. 2020;184:101718. http://dx.doi.org/10.1016/j.pneurobio.2019.101718. PMid:31669185.

7. De Nil LF, Kroll RM, Houle S. Functional neuroimaging of cerebellar activation during single word reading and verb generation in stuttering and nonstuttering adults. Neurosci Lett. 2001;302(2-3):77-80. http:// dx.doi.org/10.1016/S0304-3940(01)01671-8. PMid:11290391.

8. Brown S, Ingham RJ, Ingham JC, Laird AR, Fox PT. Stuttered and fluent speech production: an ALE meta-analysis of functional neuroimaging studies. Hum Brain Mapp. 2005;25(1):105-17. http:// dx.doi.org/10.1002/hbm.20140. PMid:15846815.

9. Chang SE, Erickson KI, Ambrose NG, Hasegawa-Johnson MA, Ludlow CL. Brain anatomy differences in childhood stuttering. Neuroimage. 2008;39(3):1333-44. http://dx.doi.org/10.1016/j.neuroimage.2007.09.067. PMid:18023366.

10. Chang SE, Zhu DC, Choo AL, Angstadt M. White matter neuroanatomical diferences in young children who stutter. Brain. 2015;138(Pt 3):694711. http://dx.doi.org/10.1093/brain/awu400. PMid:25619509.

11. Chang SE, Angstadt M, Chow HM, Etchell AC, Garnett EO, Choo $\mathrm{AL}$, et al. Anomalous network architecture of the resting brain in children who stutter. J Fluency Disord. 2018;55:46-67. http://dx.doi. org/10.1016/j.jfludis.2017.01.002. PMid:28214015.

12. Garnett EO, Chow HM, Nieto-Castañon A, Tourville JA, Guenther $\mathrm{FH}$, Chang SE. Anomalous morphology in left hemisphere motor and premotor cortex of children who stutter. Brain. 2018;141(9):2670-84. http://dx.doi.org/10.1093/brain/awy199. PMid:30084910.

13. Frigerio-Domingues CE, Gkalitsiou Z, Zezinka A, Sainz E, Gutierrez $\mathrm{J}$, Byrd C, et al. Genetic factors and therapy outcomes in persistent 
developmental stuttering. J Commun Disord. 2019;80:11-7. http:// dx.doi.org/10.1016/j.jcomdis.2019.03.007. PMid:31003007.

14. Block S, Onslow M, Packman A, Dacakis G. Connecting stuttering management and measurement: IV. Predictors of outcome for a behavioural treatment for stuttering. Int J Lang Commun Disord. 2006;41(4):395-406. http://dx.doi.org/10.1080/13682820600623853. PMid: 16815808 .

15. Perez HR, Stoeckle JH. Stuttering: clinical and research update. Can Fam Physician. 2016;62(6):479-84. PMid:27303004.

16. Yairi E, Ambrose N. Epidemiology of stuttering: 21 st century advances. J Fluency Disord. 2013;38(2):66-87. http://dx.doi.org/10.1016/j. jfludis.2012.11.002. PMid:23773662.

17. Van Beijsterveldt CE, Felsenfeld S, Boomsma DI. Bivariate genetic analyses of stuttering and nonfluency in a large sample of 5-year-old twins. J Speech Lang Hear Res. 2010;53(3):609-19. http://dx.doi. org/10.1044/1092-4388(2009/08-0202). PMid:20029049.

18. Wittke-Thompson JK, Ambrose N, Yairi E, Roe C, Cook EH, Ober $\mathrm{C}$, et al. Genetic studies of stuttering in a founder population. J Fluency Disord. 2007;32(1):33-50. http://dx.doi.org/10.1016/j.jfludis.2006.12.002. PMid:17276504.

19. Frigerio-Domingues CE, Olivera CM, Oliveira BV, Juste FS, Andrade $\mathrm{CR}$, Giacheti CM, et al. A genetic linkage study in Brazil identifies a new locus for persistent developmental stuttering on chromosome 10. Genet Mol Res. 2014;13(1):2094-101. http://dx.doi.org/10.4238/2014. March.24.13. PMid:24737434.

20. Lan J, Song M, Pan C, Zhuang G, Wang Y, Ma W, et al. Association between dopaminergic genes (SLC6A3 and DRD2) and stuttering among Han Chinese. J Hum Genet. 2009;54(8):457-60. http://dx.doi. org/10.1038/jhg.2009.60. PMid:19590515.

21. Kang C, Domingues BS, Sainz E, Frigerio-Domingues CE, Drayna D, Moretti-Ferreira D. Evaluation of the association between polymorphisms at the DRD2 locus and stuttering. J Hum Genet. 2011;56(6):472-3. http://dx.doi.org/10.1038/jhg.2011.29. PMid:21390039.
22. Frigerio-Domingues CE, Drayna D. Genetic contributions to stuttering: the current evidence. Mol Genet Genomic Med. 2017;5(2):95-102. http://dx.doi.org/10.1002/mgg3.276. PMid:28361094.

23. Kang C, Riazuddin S, Mundorff J, Krasnewich D, Friedman P, Mullikin $\mathrm{JC}$, et al. Mutations in the lysosomal enzyme-targeting pathway and persistent stuttering. N Engl J Med. 2010;362(8):677-85. http://dx.doi. org/10.1056/NEJMoa0902630. PMid:20147709.

24. Kefalianos E, Onslow M, Packman A, Vogel A, Pezic A, Mensah F, et al. The history of stuttering by 7 years of age: follow-up of a prospective community cohort. J Speech Lang Hear Res. 2017;60(10):2828-39. http://dx.doi.org/10.1044/2017_JSLHR-S-16-0205. PMid:28979988.

25. Leech KA, Bernstein-Ratner N, Brown B, Weber CM. Preliminary evidence that growth in productive language differentiates childhood stuttering persistence and recovery. J Speech Lang Hear Res. 2017;60(11):3097-109. http://dx.doi.org/10.1044/2017_JSLHR-S-16-0371. PMid:29049493.

26. Andrade CRF. Protocolos. In: Andrade CRF, editor. Gagueira infantil: risco, diagnóstico e programas terapêuticos. Barueri: Pró-Fono; 2006.p. $133-40$.

27. Andrade CRF. Fluência (Parte C). In: Andrade CRF, Befi-Lopes DM, Fernandes FDM, Wertznez HF, editores. ABFW - teste de linguagem infantil nas áreas de fonologia, vocabulário, fluência e pragmática. Barueri: Pró-Fono; 2004. p. 51-81.

28. Clark CE, Conture EG, Frankel CB, Walden TA. Communicative and psychological dimensions of the KiddyCAT. J Commun Disord. 2012;45(3):223-34. http://dx.doi.org/10.1016/j.jcomdis.2012.01.002. PMid:22333753.

29. Vanryckeghem M, Brutten GJ, Hernandez LM. A comparative investigation of the speech-associated attitude of preschool and kindergarten children who do and do not stutter. J Fluency Disord. 2005;30(4):307-18. http://dx.doi.org/10.1016/j.jfludis.2005.09.003. PMid:16246410.

30. Walsh B, Mettel KM, Smith A. Speech motor planning and execution deficits in early childhood stuttering. J Neurodev Disord. 2015;7(1):27. http://dx.doi.org/10.1186/s11689-015-9123-8. PMid:26300988. 